\title{
Diabetic ketoacidosis in children newly diagnosed with type 1 diabetes mellitus: Role of demographic, clinical, and biochemical features along with genetic and immunological markers as risk factors. A 20-year experience in a tertiary Belgian center
}

\author{
Alfredo Vicinanza $^{1,2}$ @ | Anissa Messaaoui ${ }^{1} \odot$ | Sylvie Tenoutasse ${ }^{1}$ | Harry Dorchy ${ }^{1}$
}

\author{
${ }^{1}$ Diabetology Clinic, Hôpital Universitaire des \\ Enfants Reine Fabiola, Université Libre de \\ Bruxelles, Brussels, Belgium \\ ${ }^{2}$ Pediatric Intensive Care Department, Hôpital \\ Universitaire des Enfants Reine Fabiola, \\ Université Libre de Bruxelles, Brussels, \\ Belgium

\section{Correspondence} \\ Alfredo Vicinanza, Diabetology Clinic, Hôpital \\ Universitaire des Enfants Reine Fabiola, \\ Université Libre de Bruxelles, Brussels, \\ Belgium. \\ Email: vicinanza.alfredo@gmail.com
}

\begin{abstract}
Background: Diabetic ketoacidosis (DKA) is the leading cause of morbidity and mortality in children with type 1 diabetes (T1D). Little is known about the association between genetic and immunological markers and the risk for DKA at onset of T1D. The aim of this study was to create a model foreseeing the onset of DKA in newly diagnosed patients.

Methods: This retrospective study included 532 T1D children (aged $<18$ years at diagnosis) recruited in our hospital, from 1995 to 2014. DKA and its severity were defined according to the criteria of ISPAD. Genetic risk categories for developing T1D were defined according to the Belgian Diabetes Registry. Multivariate statistical analyses were applied to investigate risk factors related to DKA at diagnosis.

Results: Overall $42 \%$ of patients presented DKA at diagnosis. This study outlined the major risk of DKA at diagnosis for younger children ( $<3$ years) and for those belonging to ethnic minorities. Children carrying neutral genotypes had a 1.5-fold increased risk of DKA at diagnosis than those with susceptible or protective genotypes, a paradoxical observation not previously reported. Only solitary positive IA-2A increased the risk of DKA at diagnosis. The proposed model could help to predict the probability of DKA in $70 \%$ of newly diagnosed cases.

Conclusions: This was the first reported implication of IA-2A positivity and neutral genotypes predisposing to DKA at diagnosis regardless of its severity. Earlier diagnosis through genetic and immunological screening of high-risk children could decrease DKA incidence at diabetes onset.
\end{abstract}

ABBREVIATIONS: AAb, autoantibodies; BA, Black Africans; BDR, Belgian Diabetes Registry; $\mathrm{BMI}$, Body Mass Index; $\mathrm{Cl}$, confidence interval; DKA, diabetic ketoacidosis; EC, European Caucasians; FDR, first-degree relative; GADA, glutamic acid decarboxylase antibodies; GCS, Glasgow Coma Scale; HbA1c, hemoglobin A1c; HLA, human leukocyte antigen; IAA, insulin autoantibodies; IA-2A, insulinoma-associated protein 2 antibodies; ICA, islet cell antibodies; IQR, interquartile range; MC, Mograbin Caucasians; MO, mixed origin; OR, odds ratio; SDR, second-degree relative; SDS, SD score; T1D, type 1 diabetes; T2D, type 2 diabetes; $\mathrm{X}$, 0201-0303, 0100-0503/0601, 0100-0503/0602/0603, 0501-0301 or 0201-0201; Y, 0100-0501/0604/0605, 0102-0201, 0102-0502, 0301-0201, 0301-0301, 0301-0303, 0301-0401, 0401-0402 or 0501-0302; yr, years; ZnT8A, zinc transporter 8 antibodies.

This is an open access article under the terms of the Creative Commons Attribution License, which permits use, distribution and reproduction in any medium, provided the original work is properly cited.

(c) 2019 The Authors. Pediatric Diabetes published by John Wiley \& Sons Ltd. 


\section{KEYWORDS}

autoantibodies, children, diabetic ketoacidosis, HLA antigens, type 1 diabetes mellitus

\section{1 | INTRODUCTION}

Diabetic ketoacidosis (DKA) is a potentially life-threatening acute complication of type 1 diabetes (T1D) characterized by a biochemical triad of hyperglycemia, ketonemia (ketonuria), and acidemia.

Diabetic ketoacidosis is still the leading cause of morbidity and mortality in children with T1D. Mortality is predominantly related to cerebral edema. DKA at T1D diagnosis results in morphologic and functional brain changes. ${ }^{1,2}$ There is a wide geographic variation in the frequency of DKA at T1D onset ${ }^{3,4}$; rates vary from $14.7 \%$ (Denmark) to $79.8 \%$ (Saudi Arabia) ${ }^{5}$ and inversely correlate with the regional incidence of the disease. ${ }^{3,4}$

A decreasing rate of DKA at diagnosis has been observed over time. ${ }^{6}$ Increasing medical information and awareness might have resulted in changes in the clinical presentation of T1D in developed countries. ${ }^{3,7,8}$

Previous studies have shown different risk factors that might increase the likelihood of presenting with DKA. ${ }^{8-10}$ Risk factors for DKA in newly diagnosed cases include: younger age $[<2$ years (yr) old], ethnic minority, lower Body Mass Index (BMI), delayed diagnosis as well as lower socioeconomic status. Protective factors are: having a first-degree relative (FDR) with T1D at the time of diagnosis, higher parental education, and higher background incidence of T1D. ${ }^{8,11}$

Specific human leukocyte antigen (HLA) class II gene alleles at the HLA-DRB1, DQA1, and DQB1 loci are the major genetic determinants for predisposition and resistance to T1D. In Caucasian populations, the highest risk for developing the disease has been associated with the DR3-DQA1*0501-DQB1*0201/DR4-DQA1*0301-DQB1*0302 genotype that increases 20 -fold the risk for T1D. ${ }^{12-14}$ In Belgium, the Belgian Diabetes Registry (BDR) has shown that this high-risk genotype confers a 21.5 -fold increased relative risk of developing diabetes before the age of $40 .^{15}$

In 1986, Ludvigsson et al. noted that DR3 patients had more often a mild disease with less ketonuria at diagnosis in North America and Europe. ${ }^{16}$ Currently, little is known about the association between various HLA class II genotypes and the risk of DKA at T1D onset. ${ }^{17,18}$

Islet autoantibodies (AAb) are also useful preclinical markers for risk of developing T1D, including: islet cell antibody (ICA), insulin autoantibody (IAA), glutamic acid decarboxylase antibody (GADA), insulinoma-associated protein 2 antibody (IA-2A), and, recently, zinc transporter 8 antibody (ZnT8A). Several prospective studies screened for islet AAb to identify children at high risk for T1D. ${ }^{19,20}$ Nevertheless, very few studies have outlined the association between $A A b$ and DKA in newly diagnosed children with T1D, most of them suggesting an almost identical prevalence of the four classical AAb in patients with T1D with or without DKA at diagnosis. ${ }^{21}$
To our knowledge, no other study has simultaneously investigated, in a large pediatric population, the association between genetic markers (HLA-DQ), AAb, age, gender, ethnicity, symptoms of T1D, glycaemia, HbA1c, C-peptide, BMI, FDR or second-degree relative (SDR) affected by T1D or type 2 diabetes (T2D) and the frequency of DKA with its severity at onset of T1D.

Therefore, the aim of this retrospective study was to analyze the effect of these different factors on ketoacidosis at T1D diagnosis and to create a multivariable model foreseeing the onset of DKA in children with newly recognized diabetes in a multi-ethnic environment like in Belgium. The ultimate purpose being to use the model in order to decrease the incidence of DKA at diabetes onset in high-risk children.

\section{2 | METHODS}

\section{1 | Subjects}

A retrospective study was conducted on a cohort including 556 children (age < 18 years at diagnosis), newly diagnosed with T1D and recruited at the Diabetology Clinic of the University Children's Hospital Queen Fabiola in Brussels, from January 1995 to December 2014; 24 patients were excluded because of too many missing values.

\subsection{Clinical and biological assessments}

A retrospective review of patients' files was analyzed in order to collect data at diagnosis.

Age at onset of T1D was expressed in years.

Ethnicity was self-reported and determined on the basis of the country of origin of the parents (European Caucasians [EC], Mograbin Caucasians [MC], Black Africans [BA], Mixed Origin [MO], other origins [Turks, Pakistanis, Latin Americans]). Moreover, the entire population was divided in two subcategories: ethnic majority including EC and $\mathrm{MC}$ and ethnic minority including $\mathrm{BA}, \mathrm{MO}$, and other origins.

Body Mass Index (BMI) was calculated in children older than 2 years, based on height and weight according to the 1990 British growth reference by Cole et al. ${ }^{22}$ In children below age $2(n=34)$, BMI was considered a missing value because of the lack of standardized age- and gender-adjusted BMI data. BMI was converted to SD scores (SDS).

Glycaemia was measured with the hexokinase method using photometric analysis modules (Cobas 8000, Roche Diagnostics, Indianapolis).

Hemoglobin $\mathrm{A} 1 \mathrm{c}(\mathrm{HbA} 1 \mathrm{c})$ level was measured with the high performance liquid chromatography system (HA-8160, Menarini Diagnostics International, Florence, Italy). 
Random C-peptide level was measured by time-resolved fluorescence immunoassay, in order to detect a residual $\beta$-cell function. The detection limit of the test was $0.2 \mu \mathrm{g} / \mathrm{L}$.

Diabetic ketoacidosis (DKA) and its severity were defined according to the criteria of the International Society for Pediatric and Adolescent Diabetes (ISPAD). ${ }^{23,24}$ As from 2004, qualitative $\beta$-ketone levels were obtained by capillary blood and analyzed with GlucoMen LX $\beta$-ketone sensor test strips by GlucoMen LX plus meter (Menarini Diagnostics). The measurement range was 0.1 to $8.0 \mathrm{mmol} / \mathrm{L}$. The $\beta$-ketone test was considered positive if $>0.6 \mathrm{mmol} / \mathrm{L}$. Ketonuria was assessed with Keto-Diabur-Test 5000 reagent strips (Roche Diagnostics, Mannheim, Germany) before the first insulin injection.

Classic symptoms of diabetes (polyurodipsia, tiredness, and weight loss) and their duration (expressed in weeks) were self-reported by patients or their parents.

Neurologic status was evaluated by Glasgow Coma Scale (GCS) scores assessed at admission and by 3 to 6 hours thereafter. Brain injuries were excluded if GCS was $\geq 14$ and in the absence of other clinical signs of cerebral edema. ${ }^{24}$ Data on the possible deterioration of neurologic status were significantly missing.

Information on the family history of T1D and T2D was obtained by interviewing the parents.

Diabetes-associated AAb. Diabetes-associated AAb, and particularly IAA, were measured before insulin administration to avoid false positive IAA results, and analyzed by the BDR. ICA was measured with an indirect immunofluorescence assay and was expressed as Juvenile Diabetes Foundation (JDF) units with cut-off values for positivity $\geq 12$ JDF units. GADA, IAA, and IA-2A were determined by liquid-phase radiobinding assays and their levels were expressed as percentages of added tracer bound (10 $000 \mathrm{cpm} /$ tube). Cut-off values were amounted to $\geq 2.6 \%$ tracer bound for GADA, $\geq 0.6 \%$ for IAA, and $\geq 0.44 \%$ tracer bound for IA-2A. During the study, the BDR did not check routinely the $\mathrm{ZnT8A}$, which was recently identified as a risk marker of T1D. ${ }^{25}$ Data for diabetes-associated autoantibodies were missing for only seven patients.

\section{3 | HLA-genotyping and associated risk categories}

Blood samples were collected within the first days of diagnosis and sent to the BDR for identification of HLA-genotyping. HLA-DQ genotype was determined using polymerase chain reaction and oligotyping with allele specific probes as described previously. ${ }^{16}$ In 22 patients, no sample was available for analysis; however, they had one or more positive diabetes-associated AAb reflecting $\beta$-cell autoimmunity. HLAassociated risk categories for developing T1D were defined according to the results reported by a genetic risk assessment Belgian study. ${ }^{14}$ As susceptible genotypes were considered: 0301-0302/0501-0201, 0301-0302/0301-0302, 0501-0201/0501-0201, and 0301-0302/Y $(\mathrm{Y}=$ 0100-0501/0604/0605, 0102-0201，0102-0502, 0301-0201, 0301-0301, 0301-0303, 0301-0401, 0401-0402, or 0501-0302); as neutral genotypes: 0501-0201/Y, 0301-0302/X (X=0201-0303, 0100-0503/0601, 0100-0503/0602/0603, 0501-0301, or
0201-0201) and $\mathrm{Y} / \mathrm{Y}$; as protective genotypes: 0501-0201/X, X/Y and $\mathrm{X} / \mathrm{X}$.

All procedures of this study were approved by the Ethics Committee of Human Experimentation in our institution and the reported investigations were carried out in accordance with the principles of the Declaration of Helsinki as revised in 2008.

\subsection{Statistical analysis}

Univariate, bivariate, and multivariate analysis were performed on our data in order to investigate risk factors related to DKA and its severity at diagnosis.

Quantitative parameters were described by their means and standard deviations or medians and interquartile ranges (IQR), qualitative parameters as frequencies and percentages.

In bivariate analysis, continuous factors were tested using Student's $t$ tests or ANOVA for equality of means and Mann-Whitney $U$ tests for equality of medians. Categorical factors were performed with $\chi^{2}$ or Fisher's exact tests of independence.

A multivariable logistic regression was performed in order to investigate the effect of multiple risk factors on children presenting with DKA at T1D onset and the results were reported as odds ratio $(\mathrm{OR})$ and $95 \%$ confidence interval $(\mathrm{Cl})$.

Statistical analysis were performed at a $5 \%$ level of significance. Results were considered statistically significant when $P$ value was $<0.05$. Statistical analysis were performed using SAS 9.2. Software.

\section{3 | RESULTS}

\section{1 | Demographic and clinical characteristics of the total population (data not shown)}

Data were available for 532 children diagnosed with new-onset T1D below the age of 18 years (boys, $n$ [\%] = 279 [52]).

Among the 532 subjects, the ethnic majority (EC and $\mathrm{MC}$ ) was composed of a total of 473 patients (89\%), whereas the ethnic minority (BA, MO, and other origins) was represented by 59 patients (11\%).

The mean age at diagnosis was 7.8 years (range: $0.2-17.5$ ); $15 \%$ were aged 0 to 3 years.

The median duration of the symptoms was 2 weeks (range: 1-3).

\subsection{Comparison of characteristics between groups with and without DKA}

Overall $42 \%$ of patients presented DKA at diagnosis, $51 \%$ of whom were boys, and $49 \%$ were girls, giving a male-to-female ratio of 1:1.

Ethnic minorities presented with DKA significantly more frequently than ethnic majorities $(P=.004)$ (Table 1). Moreover there was no difference in the occurrence of DKA between EC and MC ( $P=.335$, data not shown).

The prevalence of DKA was significantly higher in the 0 to 3 age group compared to children over 3 years $(P=.001)$ (Table 1$)$. 
TAB LE 1 Comparison of characteristics between groups with and without diabetic ketoacidsis (DKA) at diagnosis; frequencies with percentages for qualitative variables and means with standard deviations (SD) or medians with interquartile ranges (IQR) for quantitative variables

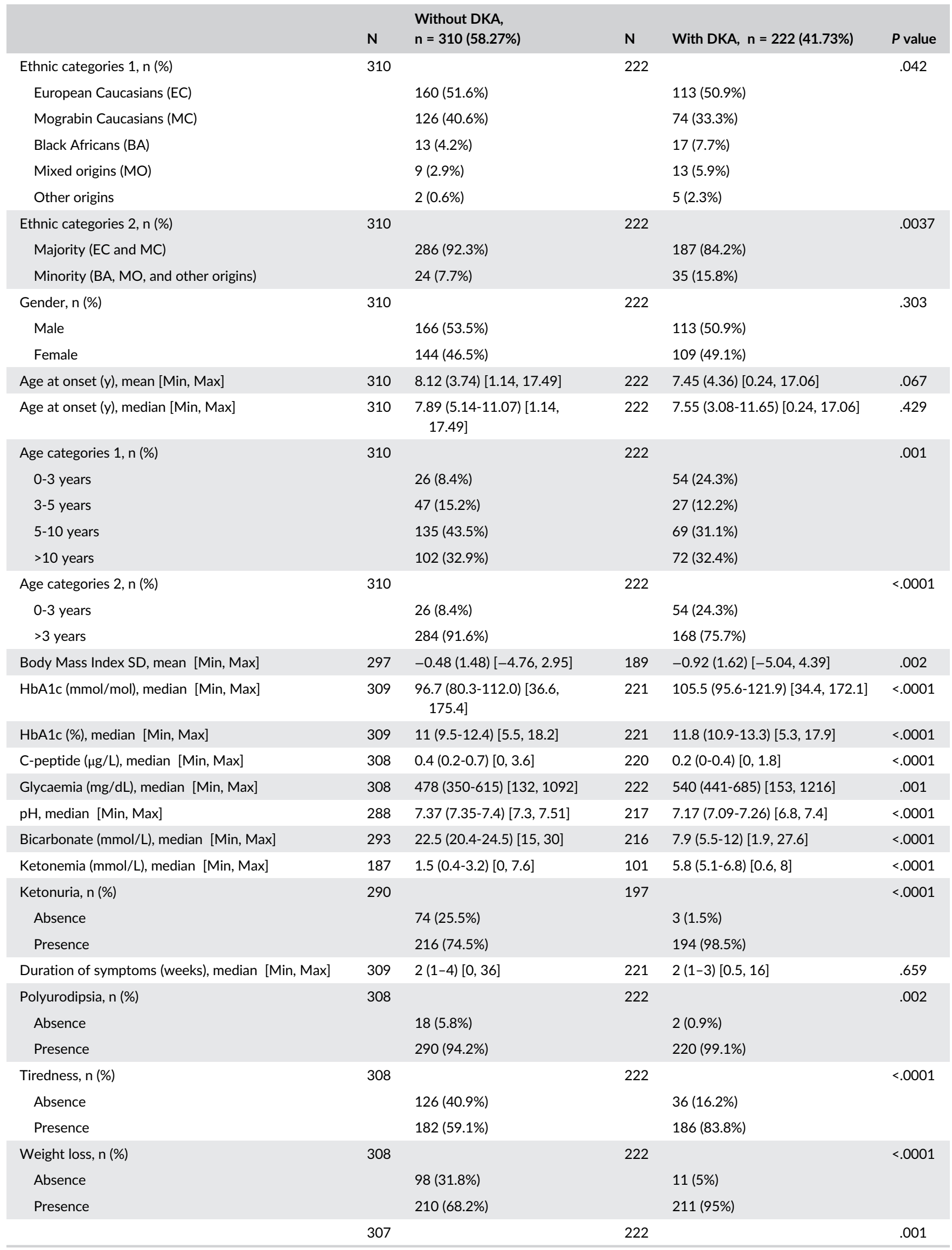


TABLE 1 (Continued)

\begin{tabular}{|c|c|c|c|c|c|}
\hline & $\mathbf{N}$ & $\begin{array}{l}\text { Without DKA, } \\
n=310(58.27 \%)\end{array}$ & $\mathbf{N}$ & With DKA, n = 222 (41.73\%) & $P$ value \\
\hline \multicolumn{6}{|l|}{$\begin{array}{l}\text { First-degree relatives (FDR) with Type } 1 \text { Diabetes } \\
\text { (T1D), } \mathrm{n}(\%)\end{array}$} \\
\hline Absence & & $266(86.6 \%)$ & & $212(95.5 \%)$ & \\
\hline Presence & & $41(13.4 \%)$ & & $10(4.5 \%)$ & \\
\hline Second-degree relatives (SDR) with T1D, n (\%) & 307 & & 222 & & .386 \\
\hline Absence & & $258(84 \%)$ & & $193(86.9 \%)$ & \\
\hline Presence & & $49(16 \%)$ & & $29(13.1 \%)$ & \\
\hline Relatives with Type 2 Diabetes (T2D), n (\%) & 306 & & 222 & & .215 \\
\hline Absence & & $178(58.2 \%)$ & & $141(63.5 \%)$ & \\
\hline Presence & & 128 (41.8\%) & & 81 (36.5\%) & \\
\hline
\end{tabular}

In children with DKA, the median $\mathrm{HbA1c}$ was significantly higher ( 105.5 vs $96.7 \mathrm{mmol} / \mathrm{mol}$ or 11.8 vs $11.0 \%, P<.0001$ ), as well as the median glycemia (540 vs $478 \mathrm{mg} / \mathrm{dL}, P=.001$ ), whereas the median random C-peptide level was significantly lower $(0.2$ vs $0.4 \mu \mathrm{g} / \mathrm{L}$, $P<.0001)$, as was the mean BMI SDS $(-0.92$ vs $-0.48, P=.002)$ at diagnosis, than in children without DKA (Table 1).

Moreover, DKA occurred less frequently in children with a FDR affected by T1D compared to children not having a FDR with T1D $(P=.001)$ (Table 1).

\subsection{Comparison of characteristics within the group of children with DKA, according to the severity of DKA (data not shown)}

Children with DKA at diagnosis ( $[\%]=222$ [42]) were divided in subcategories according to the degree of acidosis at onset (mild, moderate or severe DKA, as defined by the criteria of ISPAD ${ }^{23,24}$ ) and all the above variables were compared among the groups.

Severe form of DKA was observed in $29 \%$ of children presenting with DKA at diagnosis.

When considering ethnic minorities and majorities, there were no differences in frequencies of degrees of DKA.

The three groups differed significantly for mean age at diagnosis; the lower the age, the more severe the DKA $(P=.009)$. Above all, children below 3 years presented with severe DKA statistically more frequently than older children $(P=.026)$.

The GCS scores never declined to less than 14 and no other clinical signs of cerebral edema were reported.

No deaths linked to DKA occurred in patients with DKA of any degree at diagnosis.

\section{4 | Autoantibodies and HLA-associated risk genotypes in children with and without DKA at diagnosis and according to the severity of DKA}

Only single positive IAA had significantly higher frequencies in children with DKA compared to children without DKA at diagnosis
$(P=$.024). No solitary positive AAb were significantly associated to any degree of DKA (data not shown).

There were no significant differences in the prevalence of any multiple AAb positivity at diagnosis neither concerning the occurrence of DKA nor its severity (data not shown).

No statistically significant differences were found in the prevalence of HLA risk genotypes neither in the occurrence of DKA nor in its degrees of severity (data not shown).

Neutral genotypes had significantly higher prevalence in the ethnic minority group whether the ethnic majority group carried more frequently susceptible or protective genotypes $(P=.001)$ (Figure 1 ).

\section{5 | Multivariable logistic regression model}

This model aimed to define the predictive markers for DKA at T1D onset (Figure 2).

A significant association was observed between DKA and lower age ( $<3$ years) at diagnosis.

Children having a FDR affected by T1D had less chances of presenting with DKA at onset. Furthermore, belonging to ethnic minority groups was significantly associated with an increased risk of developing DKA.

Interestingly, carrying a neutral risk genotype was identified as an independent predictor of DKA at diagnosis as was solitary IA-2A positivity.

\section{4 | DISCUSSION}

To our knowledge, this was the first population-based analysis of children aged <18 years newly diagnosed with T1D, in which the combination of demographic, clinical, and biochemical parameters, HLA class II genotypes, autoimmune markers, and the presence of DKA at T1D onset was explored.

In this study, infants below 3 years of age showed higher frequency of DKA than older children. This finding is in line with several studies. $^{12,26}$ Furthermore, children below 3 years had 1.9-fold 


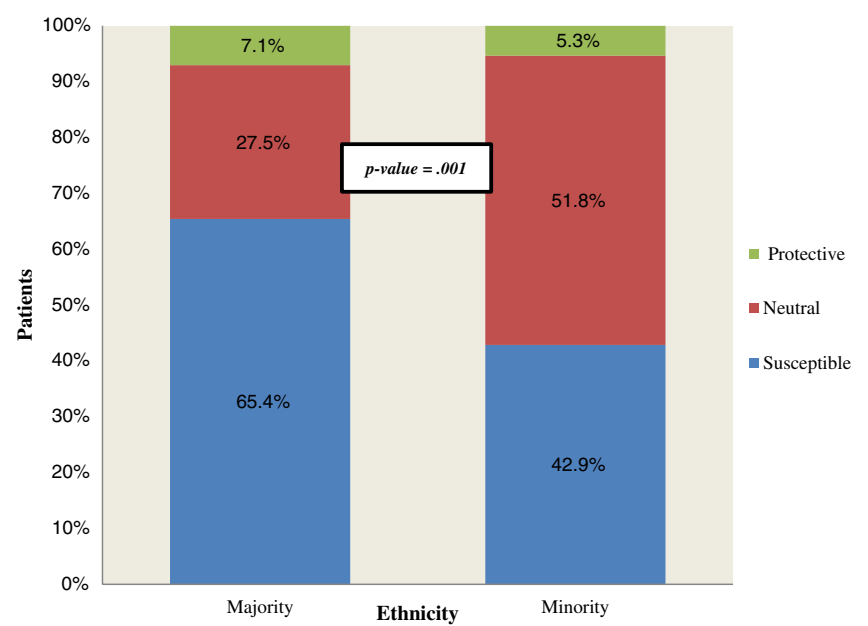

FIGURE 1 HLA risk genotypes (protective, neutral, and susceptible) by ethnicity

increased risk of severe DKA at diagnosis compared to older children (data not shown).

Neu et al. ${ }^{27}$ and Szypowska and Skórka ${ }^{10}$ suggested that children under 2 years of age remain the most prone to DKA. The short duration of symptoms and high frequency and severity of DKA in this age group have been attributed to a more rapid decline in $\beta$-cell function, further aggravated by higher incidence of intercurrent infections precipitating acidosis, higher risk of dehydration, and less developed mechanism of metabolic compensation. ${ }^{28}$

The prevalence of DKA in boys and girls was the same in our study, as already shown in a systematic review by Usher-Smith et al. ${ }^{11}$

Ketoacidosis is often considered to be the result of a late diagnosis. ${ }^{29}$ However, in our study, the duration of symptoms was similar
( 2 weeks) in children with and without DKA at presentation and at any degree of acidosis.

As expected, children presenting with DKA at diagnosis had higher glycaemia and $\mathrm{HbA} 1 \mathrm{c}$ and lower random $\mathrm{C}$-peptide level, related to poorer residual endogenous insulin secretion, as previously reported. ${ }^{30,31}$ Although well-established data revealed that a precocious diagnosis preserves the residual endogenous insulin secretion during a longer period, ${ }^{31}$ it has been shown that early intensive therapy did not provide benefits in preserving $\beta$-cell function. ${ }^{32}$ Messaaoui et al. showed that younger age and lower C-peptide level at T1D onset are correlated with an increased glomerular filtration rate which could be responsible for later nephropathy. ${ }^{33}$

Like other authors, ${ }^{8,18}$ we also observed that having a FDR with T1D is a preventing factor against DKA at diagnosis.

Our results highlighted the major risk of presenting with DKA at diagnosis for children belonging to ethnic minorities (BA, MO, and other origins) compared to ethnic majorities (EC and $\mathrm{MC}$ ), as already reported. ${ }^{15,34}$ Youth of minority race/ethnicity have increased markers of poor prognosis of type 1 diabetes at diagnosis and 3 years postdiagnosis. ${ }^{35}$ Ethnic minority status is also a consistent indicator of risk for DKA in children and adolescents with previously diagnosed T1D. ${ }^{36}$

When $A A b$ variables were introduced in the logistic regression model, only solitary positive IA-2A increased the risk of the occurrence of DKA at diagnosis regardless of its severity, while all the other single positive $A A b$ were no longer significant; this result has here been observed for the first time. The BDR showed that IA-2A has a higher positive predictive value as a marker of T1D than other AAb. Moreover, the prevalence of $I A-2 A$ is higher in children than in adults. ${ }^{37}$ It has been suggested that islet AAb screenings may lead to

\section{Odds Ratios with $\mathbf{9 5 \%}$ Wald Confidence Limits}

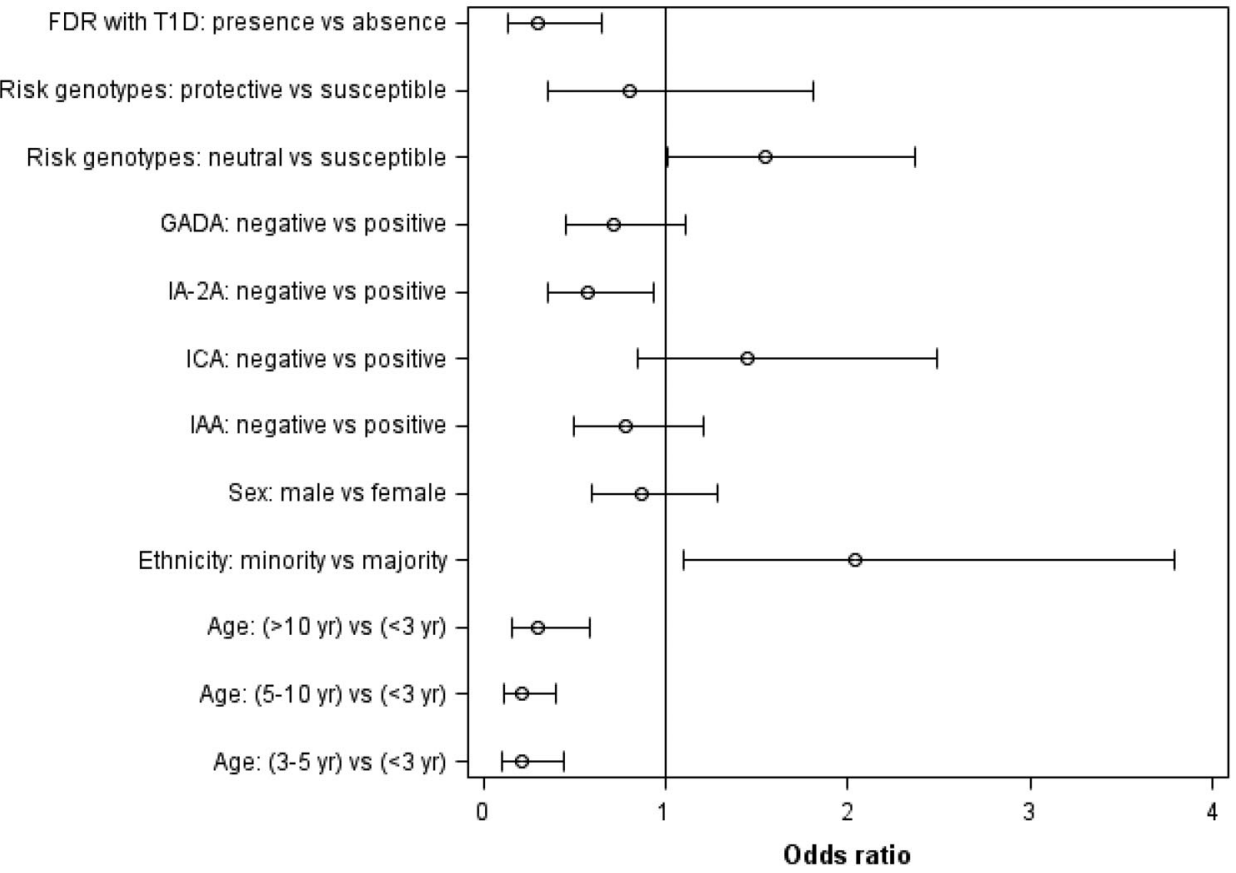

FIGURE 2 Multivariate analysis of risk factors for diabetic ketoacidosis (DKA) at diagnosis. The dot denotes the estimated odds ratios (OR) from the logistic regression model and the bar denotes the $95 \%$ confidence interval. FDR, first-degree relative; GADA, glutamic acid decarboxylase antibodies; IA-2A, insulinoma-associated protein 2 antibodies; ICA, islet cell antibodies; IAA, insulin autoantibodies 
an earlier diagnosis of T1D and, thereby, prevent DKA at onset and improve the clinical course after diagnosis. ${ }^{19,21}$

The nature and prevalence of HLA-DQ and HLA-DR haplotypes and genotypes susceptibility for T1D may differ between populations throughout the world. ${ }^{38-40}$

In a Belgian study including 452 patients with T1D aged 0.1-17.5 years at diagnosis, the proportion of the different ethnic groups was: $55 \% \mathrm{EC}, 35 \% \mathrm{MC}, 6 \% \mathrm{BA}$, and $4 \% \mathrm{MO}$. The two most frequent haplotypes were DQA1*0501-DQB1*0201 and $D Q A 1 * 0301-D Q B 1 * 0302$ with a significant higher prevalence in MC and EC. The high-risk heterozygous genotype DQA1*0301$D Q B 1 * 0302 / D Q A 1 * 0501-D Q B 1 * 0201$ was more frequent in EC than in $M C$, whereas the homozygous genotype DQA1*0501-DQB1*0201/ DQA1*0501-DQB1*0201 was more prevalent in MC. DQA1*0301$D Q B 1 * 0201$ haplotype was found in more than half of Black African patients in the Belgian study by Stoupa and Dorchy. ${ }^{38}$ However, the size of the subgroup of BA was small, counting only 26 patients. HLADQ genotypes differ by ethnicity in patients with childhood onset T1D residing in Belgium, but not in diabetes-associated autoantibodies or other immune markers. ${ }^{38}$

Recently, a Finnish study showed that children with HLA genotypes conferring strong or moderate risk for T1D had a lower frequency of DKA and severe DKA than subjects with other HLA class II genotypes. $^{17}$

On the contrary, in an Italian study, subjects with increased HLAassociated risk genotypes were more likely to present DKA at T1D onset, compared to subjects with neutral or decreased HLAassociated risk genotypes. ${ }^{18}$

It is noteworthy that, in our study, children with neutral genotypes had a 1.5-fold increased risk of DKA at diagnosis than children with susceptible or protective genotypes, an observation not previously reported. Interestingly, neutral genotypes were more frequent in ethnic minorities and, paradoxically, these parameters were both independent predictors of DKA at T1D onset in the logistic regression analysis.

Standardized criteria for definition of DKA were proposed by ISPAD in 2014 and 2018. ${ }^{23,24}$ Irrespective of different definitions of DKA, the general observation is that the prevalence of DKA is decreasing when the incidence of T1D is increasing. ${ }^{41}$ International studies observed a continuous increase of the incidence of T1D in children. ${ }^{42}$ In Belgium, before age 40 , the incidence of type 1 diabetes averages 10 new cases per 100000 persons per year, rates increasing by $3.7 \%$ annually in children $<15$ years. The incidence rate of T1D doubled over a 15-year period in Belgian children $<15$ years and more than doubled in those $<5$ years. ${ }^{43,44}$ In our population we noted that the frequency of DKA at disease onset did not vary over time although the incidence of type 1 diabetes more than doubled during the 20-year period of observation (data not shown).

Earlier diagnosis through genetic and immunological screenings of high-risk children could decrease DKA incidence at diabetes onset. $^{45,46}$ The Environmental Determinants of Diabetes in the Young (TEDDY) study recently showed a decreased prevalence of DKA in youth $<5$ years of age participating in a longitudinal follow-up. ${ }^{47}$ In
Belgium, the BDR systematically performs genetic and annual immunological assessments in FDR of T1D patients $<40$ years.

Based on the analysis of risk factors for DKA at T1D onset discussed above, our multivariable model could help to identify high-risk children for DKA at T1D diagnosis.

Therefore, in order to prevent the incidence of DKA in newly diagnosed children, a medical follow-up, as well as parental learning to recognize early symptoms of T1D, should be carried out on mainly the following five target groups: children with a FDR affected by T1D, younger children ( $<3$ years), those belonging to ethnic minorities, children with IA-2A positivity at immunological screenings and the ones carrying neutral genotypes at the genetic assessment. In our opinion, the last two markers are original.

The ROC curve in our study showed that this model would be able to correctly predict the probability of DKA at diagnosis in almost $70 \%$ of cases (Figure 3), thus preventing admissions to pediatric intensive care unit and reducing hospital lengths of stay and costs. Of course, this outcome could be further explored by applying our model to another test population.

On a national scale, a prevention campaign should be started, aiming at increasing awareness and knowledge of the signs and symptoms of diabetes, such as the classic triad of polydipsia, polyuria, and weight loss. This could be done through initiatives in the public education system, and specific campaigns addressed to families and schools (eg, by posters and TV spots), and above all involving targeted education of health professionals. This could be an effective strategy for the early detection of type 1 diabetes and the reduction of the still relatively high prevalence of DKA in children with newly recognized diabetes in Belgium. In Italy, a school and physician awareness campaign launched by Vanelli et al. in the nineties, ${ }^{7}$ targeting children from 6 to

ROC Curve for Model

Area Under the Curve $=0.6937$

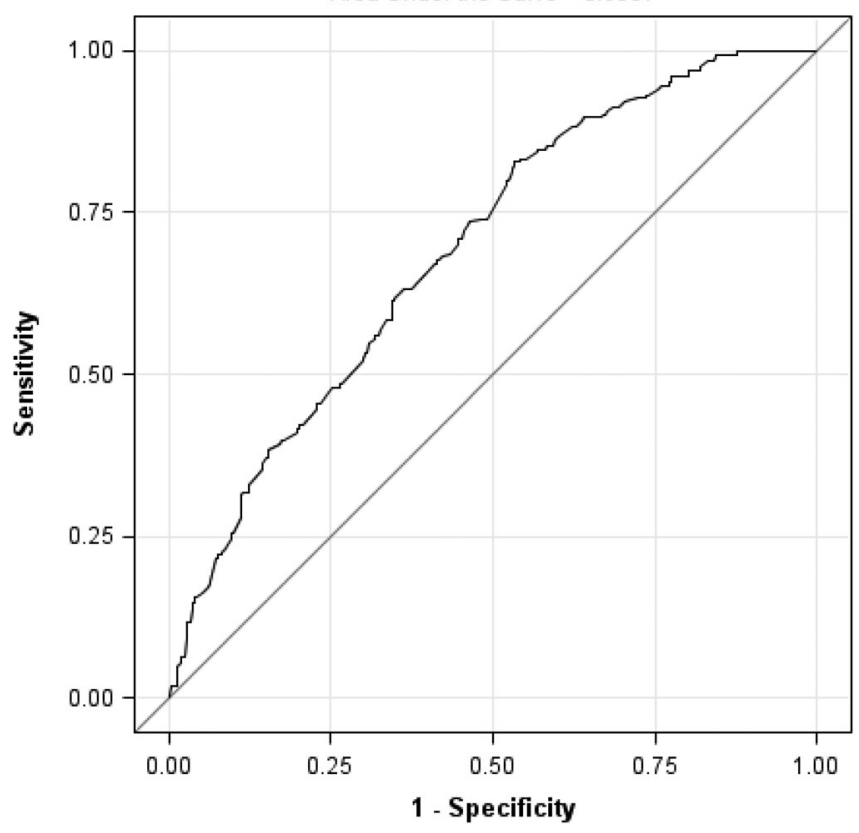

FIGURE 3 ROC curve for logistic regression model 
14 year olds, reduced the cumulative frequency of DKA at diagnosis from $78 \%$ to $13 \%$ over a 8 -year period. More recently, similar results were obtained by an information campaign in France. ${ }^{8}$ Nevertheless, a very recent once-yearly untargeted campaign to raise awareness of diabetes symptoms in youth had limited impact. ${ }^{48}$ The efficiency and cost effectiveness of strategies to reduce the incidence of DKA at T1D onset are important issues for future interventions.

In industrialized countries, the mortality rate from DKA in children is $0.15 \%$ to $0.30 \% .{ }^{49}$ Cerebral edema accounts for $60 \%$ to $90 \%$ of all DKA deaths. ${ }^{2,49}$ Despite subtle cerebral edema was doubtless underestimated in our retrospective cohort because of substantial missing data, no episodes of decline in neurologic status leading to initiation of hyperosmolar therapy or endotracheal intubation were reported during treatment for DKA. However, clinically apparent brain injury occurs in less than $1 \%$ of episodes of DKA during treatment with different rates of rehydration and fluid sodium chloride content in a clinical trial by Kuppermann et al. ${ }^{50}$ Likewise, there was no mortality linked to DKA in our cohort over 20 years. This may be attributed to a milder presentation along with the strict adherence to ISPAD as well as to our diabetology clinic DKA management protocols. $^{23,24,51}$

Although it involved a large and multi-ethnic population of children, this study had limitations. This was a single-center retrospective study; moreover, the number of patients non EC or MC was relatively small. Therefore, caution should be used in generalizing these findings to other populations.

\section{5 | CONCLUSIONS}

As DKA at diagnosis of T1D is still a cause of mortality in children with T1D, the identification of risk factors for DKA is of the utmost importance.

We confirmed that children aged $<3$ years, those without a FDR affected by T1D and ethnic minorities are at higher risk of developing DKA.

To our knowledge, this was the first reported implication of IA-2A positivity and neutral genotypes predisposing to DKA at diagnosis regardless of its severity. The proposed multivariable model could help to predict the probability of DKA in approximately $70 \%$ of newly diagnosed cases.

Prevention programs should consider the application of special measures to avoid DKA in these high-risk groups.

\section{ACKNOWLEDGEMENTS}

The authors would like to thank especially Mr Dimitri H. L. Gayraud for his contribution to the statistical analysis of the data, the Belgian Diabetes Registry for the collection of the HLA-DQ data, and of diabetes-associated autoantibodies and the whole team of Diabetology Clinic for their technical assistance.

\section{CONFLICT OF INTEREST}

The authors declare no potential conflict of interest.

\section{AUTHOR CONTRIBUTIONS}

A.V. concepted and designed the work, wrote, and drafted the manuscript, was mainly responsible for the acquisition, analysis, and interpretation of data.

A.M. and S.T. helped with data acquisition and contributed to the interpretation of results.

H.D. substantially contributed to conception and design of the work, to the acquisition, analysis, and interpretation of data, drafted the article and revised it critically for important intellectual content.

All authors critically reviewed the manuscript. All authors gave final approval of the version to be published.

\section{ORCID}

Alfredo Vicinanza (D) https://orcid.org/0000-0002-9549-3519

Anissa Messaaoui (1D https://orcid.org/0000-0002-0331-8589

\section{REFERENCES}

1. Dunger DB, Sperling MA, Acerini CL, et al. European Society for Paediatric Endocrinology/Lawson Wilkins Pediatric Endocrine Society consensus statement on diabetic ketoacidosis in children and adolescents. Pediatrics. 2004;113:133-140.

2. Cameron FJ, Scratch SE, Nadebaum C, et al. Neurological consequences of diabetic ketoacidosis at initial presentation of type 1 diabetes in a prospective cohort study of children. Diabetes Care. 2014; 37:1554-1562.

3. Levy-Marchal C, Patterson CC, Green A. Geographical variation of presentation at diagnosis of type I diabetes in children: the EURODIAB study. Diabetologia. 2001;44:75-80.

4. Usher-Smith JA, Thompson M, Ercole A, Walter FM. Variation between countries in the frequency of diabetic ketoacidosis at first presentation of type 1 diabetes in children: a systematic review. Diabetologia. 2012;55:2878-2894.

5. Große J, Hornstein H, Manuwald U, Kugler J, Glauche I, Rothe U. Incidence of diabetic ketoacidosis of New-onset type 1 diabetes in children and adolescents in different countries correlates with human development index (HDI): an updated systematic review, meta-analysis, and meta-regression. Horm Metab Res. 2018;50:209-222.

6. Hekkala A, Knip M, Veijola R. Ketoacidosis at diagnosis of type 1 diabetes in children in northern Finland: temporal changes over 20 years. Diabetes Care. 2007;30:861-866.

7. Vanelli M, Chiari G, Ghizzoni L, Costi G, Giacalone T, Chiarelli F. Effectiveness of a prevention program for diabetic ketoacidosis in children. An 8-year study in schools and private practices. Diabetes Care. 1999; 22:7-9.

8. Choleau C, Maitre J, Elie C, et al. Ketoacidosis at time of diagnosis of type 1 diabetes in children and adolescents: effect of a national prevention campaign. Arch Pediatr. 2015;22:343-351.

9. Fritsch M, Rosenbauer J, Schober E, Neu A, Placzek K, Holl RW. Predictors of diabetic ketoacidosis in children and adolescents with type 1 diabetes. Experience from a large multicenter database. Pediatr Diabetes. 2011;12:307-312. 
10. Szypowska A, Skórka A. The risk factors of ketoacidosis in children with newly diagnosed type 1 diabetes mellitus. Pediatr Diabetes. 2011;12:302-306.

11. Usher-Smith JA, Thompson MJ, Sharp SJ, Walter FM. Factors associated with the presence of diabetic ketoacidosis at diagnosis of diabetes in children and young adults: a systematic review. BMJ. 2011;343: 4092.

12. Hermann R, Turpeinen $\mathrm{H}$, Laine AP, et al. HLA DR-DQ-encoded genetic determinants of childhood-onset T1D in Finland: an analysis of 622 nuclear families. Tissue Antigens. 2003;62:162-169.

13. Nokoff N, Rewers M. Pathogenesis of type 1 diabetes: lessons from natural history studies of high-risk individuals. Ann N Y Acad Sci. 2013;1281:1-15.

14. Dorchy H, Vandewalle C, Decraene T, Nagy ZP, Schuit F, Gorus F. Genetic and immunological markers in European Caucasians and Mograbin Caucasians with type 1 (insulin-dependent) diabetes residing in Belgium. Pediatr Adolesc Endocrinol. 1993;23: 71-75.

15. Van der Auwera BJ, Schuit FC, Weets I, Ivens A, Van Autreve JE, Gorus FK. Relative and absolute HLA-DQA1-DQB1 linked risk for developing type I diabetes before 40 years of age in the Belgian population: implications for future prevention studies. Hum Immunol. 2002;63:40-50.

16. Ludvigsson J, Samuelsson U, Beauforts C, et al. HLA-DR 3 is associated with a more slowly progressive form of type 1 (insulin-dependent) diabetes. Diabetologia. 1986;29:207-210.

17. Hekkala A, llonen J, Knip M, Veijola R. Family history of diabetes and distribution of class II HLA genotypes in children with newly diagnosed type 1 diabetes: effect on diabetic ketoacidosis. Eur $J$ Endocrinol. 2011;165:813-817.

18. Marigliano M, Morandi A, Maschio M, et al. Diabetic ketoacidosis at diagnosis: role of family history and class II HLA genotypes. Eur J Endocrinol. 2012;168:107-111.

19. TEDDY Study Group. The environmental determinants of diabetes in the young (TEDDY) study: study design. Pediatr Diabetes. 2007;8: 286-298.

20. Barker JM, Goehrig SH, Barriga K, et al. Clinical characteristics of children diagnosed with type 1 diabetes through intensive screening and follow-up. Diabetes Care. 2004;27:1399-1404.

21. Ostman J, Landin-Olsson M, Törn C, et al. Ketoacidosis in young adults is not related to the islet antibodies at the diagnosis of type 1 diabetes mellitus--a nationwide study. Diabet Med. 2000;17: 269-274.

22. Cole TJ, Freeman JV, Preece MA. Body mass index reference curves for the UK, 1990. Arch Dis Child. 1995;73:25-29.

23. Wolfsdorf JI, Allgrove J, Craig ME, et al. ISPAD clinical practice consensus guidelines 2014. Diabetic ketoacidosis and hyperglycemic hyperosmolar state. Pediatr Diabetes. 2014;15:154-179.

24. Wolfsdorf JI, Glaser N, Agus M, et al. ISPAD clinical practice consensus guidelines 2018: diabetic ketoacidosis and the hyperglycemic hyperosmolar state. Pediatr Diabetes. 2018;19:155-177.

25. Vermeulen I, Weets I, Asanghanwa M, et al. Contribution of antibodies against IA-2 $\beta$ and zinc transporter 8 to classification of diabetes diagnosed under 40 years of age. Diabetes Care. 2011;34:17601765.

26. Quinn M, Fleischman A, Rosner B, Nigrin DJ, Wolfsdorf Jl. Characteristics at diagnosis of type 1 diabetes in children younger than 6 years. J Pediatr. 2006;148:366-371.

27. Neu A, Willasch A, Ehehalt S, Hub R, Ranke MB. Ketoacidosis at onset of type 1 diabetes mellitus in children - frequency and clinical presentation. Pediatr Diabetes. 2003;4:77-81.

28. Nimri R, Phillip M, Shalitin S. Children diagnosed with diabetes during infancy have unique clinical characteristics. Horm Res. 2007;67: 263-267.
29. Bui H, To T, Stein R, Fung K, Daneman D. Is diabetic ketoacidosis at disease onset a result of missed diagnosis? J Pediatr. 2010;156: 472-477.

30. Keenan HT, Foster CM, Bratton SL. Social factors associated with prolonged hospitalization among diabetic children. Pediatrics. 2002; 109:40-44.

31. Steck AK, Larsson HE, Liu X, et al. Residual beta-cell function in diabetes children followed and diagnosed in the TEDDY study compared to community controls. Pediatr Diabetes. 2017;18:794-802.

32. Buckingham B, Beck RW, Ruedy KJ, et al. Effectiveness of early intensive therapy on $\beta$-cell preservation in type 1 diabetes. Diabetes Care. 2013;36:4030-4035.

33. Messaaoui $A$, Tenoutasse $S$, Mélot $C$, Dorchy $H$. Inverse relationship between glomerular hyperfiltration and C-peptide level in type 1 diabetes. J Diabetes Mellitus. 2014;4:50-53.

34. Dabelea D, Rewers A, Stafford JM, et al. Trends in the prevalence of ketoacidosis at diabetes diagnosis: the SEARCH for diabetes in youth study. Pediatrics. 2014;133:938-945.

35. Redondo MJ, Libman I, Cheng P, et al. Racial/ethnic minority youth with recent-onset type 1 diabetes have poor prognostic factors. Diabetes Care. 2018;41:1017-1024.

36. Maahs DM, Hermann JM, Holman N, et al. Rates of diabetic ketoacidosis: international comparison with 49,859 pediatric patients with type 1 diabetes from England, Wales, the U.S., Austria, and Germany. Diabetes Care. 2015;38:1876-1882.

37. Gorus FK, Goubert P, Semakula C, et al. IA-2-autoantibodies complement GAD65-autoantibodies in new-onset IDDM patients and help predict impending diabetes in their siblings. The Belgian Diabetes Registry. Diabetologia. 1997;40:95-99.

38. Stoupa A, Dorchy H. HLA-DQ genotypes - but not immune markers differ by ethnicity in patients with childhood onset type 1 diabetes residing in Belgium. Pediatr Diabetes. 2016;17:342-350.

39. Lipton RB, Drum M, Greeley SA, Danielson KK, Bell GI, Hagopian WA. HLA-DQ haplotypes differ by ethnicity in patients with childhood-onset diabetes. Pediatr Diabetes. 2011;12:388-395.

40. Black MH, Lawrence JM, Pihoker C, et al. HLA-associated phenotypes in youth with autoimmune diabetes. Pediatr Diabetes. 2013;14: 121-128.

41. Sadauskaite-Kuehne V, Samuelsson $U$, et al. Severity at onset of childhood type 1 diabetes in countries with high and low incidence of the condition. Diabetes Res Clin Pract. 2002;55:247-254.

42. Patterson CC, Dahlquist GG, Gyürüs E, Green A, Soltész G. Incidence trends for childhood type 1 diabetes in Europe during 1989-2003 and predicted new cases 2005-20: a multicentre prospective registration study. Lancet. 2009;373:2027-2033.

43. Weets I, De Leeuw IH, Du Caju MV, et al. The incidence of type 1 diabetes in the age group 0-39 years has not increased in Antwerp (Belgium) between 1989 and 2000: evidence for earlier disease manifestation. Diabetes Care. 2002;25:840-846.

44. Weets I, Rooman R, Coeckelberghs M, et al. The age at diagnosis of type 1 diabetes continues to decrease in Belgian boys but not in girls: a 15-year survey. Diabetes Metab Res Rev. 2007;23:637-643.

45. Diabetes Prevention Trial-Type 1 Diabetes Study Group. Effects of insulin in relatives of patients with type 1 diabetes mellitus. $N$ Engl $J$ Med. 2002;346:1685-1691.

46. Decochez K, De Leeuw IH, Keymeulen B, et al. IA-2 autoantibodies predict impending type I diabetes in siblings of patients. Diabetologia. 2002;45:1658-1666.

47. Elding Larsson H, Vehik K, Bell R, et al. Reduced prevalence of diabetic ketoacidosis at diagnosis of type 1 diabetes in young children participating in longitudinal follow-up. Diabetes Care. 2011; 34:2347-2352.

48. Derraik JGB, Cutfield WS, Maessen SE, et al. A brief campaign to prevent diabetic ketoacidosis in children newly diagnosed with type 
1 diabetes mellitus: the NO-DKA study. Pediatr Diabetes. 2018;19: 1257-1262.

49. Decourcey DD, Steil GM, Wypij D, Agus MS. Increasing use of hypertonic saline over mannitol in the treatment of symptomatic cerebral edema in pediatric diabetic ketoacidosis: an 11-year retrospective analysis of mortality. Pediatr Crit Care Med. 2013;14:694-700.

50. Kuppermann N, Ghetti S, Schunk JE, et al. Clinical trial of fluid infusion rates for pediatric diabetic ketoacidosis. N Engl J Med. 2018;378: 2275-2287.

51. Tenoutasse S, Mouraux T, Dorchy H. Diabetic ketoacidosis: diagnosis, management, prevention. Rev Med Brux. 2010;31:71-76.
How to cite this article: Vicinanza A, Messaaoui A, Tenoutasse S, Dorchy H. Diabetic ketoacidosis in children newly diagnosed with type 1 diabetes mellitus: Role of demographic, clinical, and biochemical features along with genetic and immunological markers as risk factors. A 20-year experience in a tertiary Belgian center. Pediatr Diabetes. 2019; 1-10. https://doi.org/10.1111/pedi.12864 